\title{
The Current Status and a New Approach for Chinese Doctors to Obtain Medical Knowledge Using Social Media: A Study of WeChat
}

\author{
Li Liu, ${ }^{1}$ Kunyan Wei, ${ }^{2}$ Xingting Zhang $\mathbb{D}^{3},{ }^{3}$ Dong Wen $\mathbb{D},{ }^{3}$ Li Gao, ${ }^{4}$ and Jianbo Lei $\mathbb{D}^{5,6}$ \\ ${ }^{1}$ Sichuan Cancer Hospital \& Institute, Chengdu, China \\ ${ }^{2}$ Department of Gastroenterology, Affiliated Hospital of Southwest Medical University, Luzhou, China \\ ${ }^{3}$ Peking University Third Hospital, Beijing, China \\ ${ }^{4}$ Peking University School of Stomatology, Beijing, China \\ ${ }^{5}$ Center for Medical Informatics, Peking University, Beijing, China \\ ${ }^{6}$ School of Medical Informatics and Engineering, Southwest Medical University, Luzhou, China
}

Correspondence should be addressed to Jianbo Lei; jblei@hsc.pku.edu.cn

Received 23 December 2017; Revised 22 March 2018; Accepted 3 April 2018; Published 8 May 2018

Academic Editor: Haoran Xie

Copyright ( $2018 \mathrm{Li}$ Liu et al. This is an open access article distributed under the Creative Commons Attribution License, which permits unrestricted use, distribution, and reproduction in any medium, provided the original work is properly cited.

Background. WeChat is the most widely and frequently used mobile social media in China and has profoundly integrated into the daily life of many Chinese people. A variety of medicine-related information may be found on WeChat. As users of WeChat, doctors often access health-related information and even provide a variety of medical services or participate in various types of mobile communication with patients. Objective. This study is the first attempt to quantitatively explore the approaches by doctors of acquiring medical knowledge using Internet resources especially social media such as WeChat to access knowledge. Methods. A self-administered questionnaire was designed, distributed, collected, and analyzed utilizing the online survey tool Sojump. WeChat was adopted to randomly release the questionnaires using snowball sampling and collect the results after a certain amount of time. Results. 292 valid questionnaires out of 314 questionnaires by clinical doctors were analyzed. Regarding the current status of accessing medical knowledge among doctors, more than $60 \%$ of the doctors regularly used the Internet to search for medical knowledge, $19.86 \%$ used WeChat as a channel to acquire medical knowledge, and only $23.97 \%$ were satisfied with acquiring medical knowledge through the Internet. Regarding the frequency of WeChat usage, nearly $40 \%$ of the doctors accessed WeChat more than 20 times per day and over 70\% used WeChat for over half an hour every day. Regarding the status of accessing medical knowledge through WeChat, nearly half $(47.26 \%)$ of the doctors stated that they often read professional medical articles on WeChat and the most common channel is friends' moment sharing and public account subscriptions, with selection rates of 59.93\% and $60.27 \%$, respectively. The most desirable mode of acquiring medical knowledge through WeChat was the following: "professional medical knowledge from peers, with a reminder." Conclusion. WeChat has become a nonnegligible means of acquiring medical knowledge for busy Chinese physicians in a mobile environment. Further evaluation and improvement of the quality of medical knowledge on WeChat are needed. The recommendation of individualized articles through social media may become another contributing factor for doctors to acquire medical knowledge effectively and efficiently.

\section{Background}

The amount of information required for medical practice is growing at an exponential rate, and it has become less practical to completely master the tremendous amount of knowledge with doctors' individual ability [1]. Doctors are faced with the major challenge of handling a flood of professional information. The traditional learning model cannot meet the requirements of the new situation, and there is an urgent need for a new learning model to solve this dilemma [2]. The development of mobile device based social media has provided an idea for a solution $[3,4]$. Social media are tools for online learning and distance education. Narrowly speaking, a social medium is a form of discussion by users 
based on the website and application, and its main contents are generated by users, who can create, retrieve, and discover these contents [2]. In recent years, social media as learning tools have been growing rapidly in the medical field, forming an online learning mode which is learner-oriented with a relatively high degree of collaboration and participation [5].

The rapid development of the Internet and social media is profoundly changing people's lifestyles. Nearly $90 \%$ of American adults are using the Internet [6], of whom $72 \%$ access social networking websites [7]. More than 75,000 healthcare professionals around the world share information and discuss treatment options on Twitter [8]. In China, the number of mobile phone users had reached 668 million, and mobile Internet usage has reached $88.9 \%$ [9]. WeChat, a free application that provides an instant messaging service to intelligent terminals such as mobile phones launched on January 21, 2011 by Tencent [10]. Via cell phones, WeChat can integrate the functions of instantaneousness and socialization and break through temporal and geographical constraints; thus, it has become the mobile social platform with the largest number of users, the widest application, and the highest frequency in China, showing the most power of influence [11]. According to the latest official data published by Tencent, in September 2015, the number of active WeChat users had reached 570 million, covering over 200 countries in more than 20 languages [12]. WeChat has become not only a cell phone application with many innovative features but also an indispensable tool for everyday use in people's lives, covering more than $90 \%$ of China's smart mobile phones and forming a lifestyle in cell phone users [13].

As a social medium, WeChat carries a variety of topics and articles, of which many are concerning medical expertise [14-16]. Doctors are a part of the large community of WeChat users, and many researchers have noted this phenomenon. Medical education by social media has been accepted by doctors around the world [5, 17-19]. In recent years, an increasing number of studies on emerging social media in the medical field, from mobile phone texts to Twitter, Facebook, and microblogs, have been reported [20-22]. However, few quantitative studies on doctors' attitude in using social media are available in the literature [23]. A study on the top website for doctors in the US reported that many doctors seek advice from the social networking website when confronted with a difficult case and discuss health policy and medical research online [24]. However, studies on the use of social media in physicians for continuing medical education are also lacking. How social media with strong interaction and high usage such as WeChat affect the behavior, learning of medical knowledge, doctor-patient interaction, and even online medical services in physicians is an area that warrants investigation. This study targets continuing medical education and is the first to quantitatively investigate Chinese doctors' attitudes toward the channels for acquiring medical knowledge and continuing medical education using WeChat.

\section{Research Methods}

2.1. Questionnaire Design. Regarding the survey, Sojump .com (URL: https://www.sojump.com/) was used to generate, distribute, and collect the questionnaire. Sojump.com is a professional platform for online surveys, assessment, and voting; it focuses on providing a series of powerful and userfriendly services, including online questionnaire design, data collection, customized reports, and analysis of the survey results. Compared to the traditional method of investigation and other survey sites or survey systems, Sojump.com has the obvious advantages of being rapid, easy to use, and inexpensive [25].

Since there is no existing questionnaire able to answer the current status of physicians to acquire medical knowledge via social media, especially WeChat, we have to develop our own questionnaire. According to the objective of this study, a multidisciplinary team was formed to review relevant literature and discuss study needs and methods to develop the questionnaire. A final questionnaire on the knowledge, attitude, and behavior regarding WeChat and health and medical problems was designed based on the experience of previous questionnaire development and a consideration of the characteristics of social media. The survey mainly included the following framework: general demographic indicators, the usage situation of WeChat, the status of obtaining medical knowledge and health education through WeChat, the desired approach for health education, and the existing problems of healthcare and medical information on WeChat. A pilot test was conducted for the initial questionnaire after being developed by a team of three doctors and two survey experts. Based on the type of related questions, the length of the questions, the answer options, and the time to complete the questionnaire in the feedback, the questionnaire was modified and improved, resulting in the final questionnaire, which was available online at the following URL: www.sojump.com/jq/5854804.aspx.

2.2. Data Sample and Survey Methods. WeChat is a social tool with a powerful user group in China, and nearly half of its active users have more than 100 WeChat friends [12]. In this study, the questionnaires were distributed by WeChat with the snowball sampling method. From October 10, 2015 to October 21, 2015, the self-designed questionnaire for medical doctors on using WeChat to acquire medical knowledge was distributed to 2000 friends and a variety of groups belonging to the author's WeChat account (approximately 20 groups, with a minimum of eight members and a maximum of 314 members). Each receiver was asked to forward the questionnaire link to other WeChat users through friends' network on WeChat, and ultimately, 314 questionnaires were collected; of these, 292 questionnaires from the physician respondents were valid.

2.3. Data Processing. The collected questionnaires were analyzed, and the invalid questionnaires were excluded based on the following exclusion criteria: repeated IP address in the response; logical errors in the answers; the same answer for successive questions; completion time being too short; and completion time being too long. A total of 22 invalid questionnaires were excluded, resulting in 292 valid questionnaires. Data analysis was conducted using the builtin statistical function in the backend of Sojump.com. 


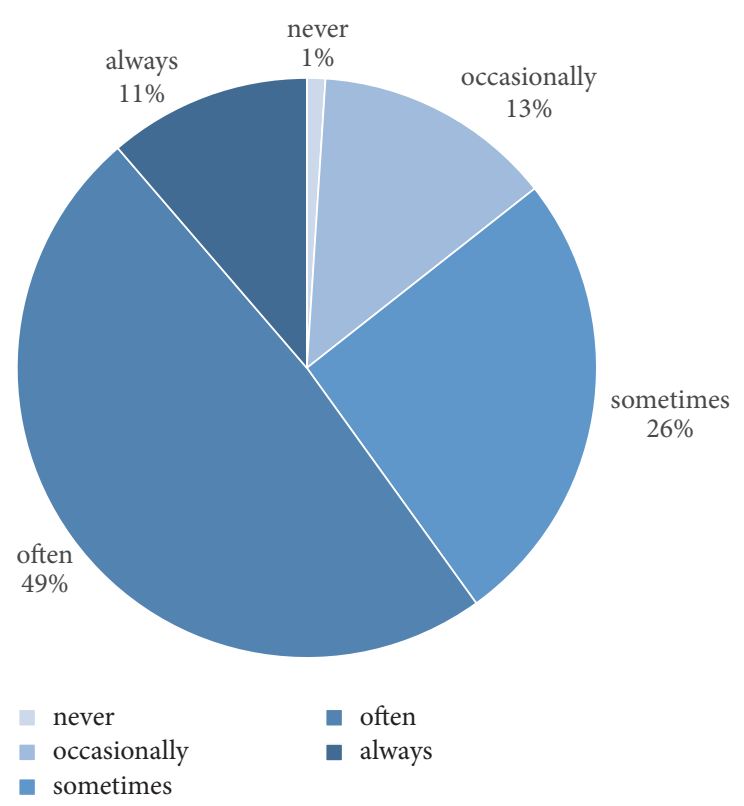

FIGURE 1: Frequency of Internet usage by doctors to search for medical knowledge.

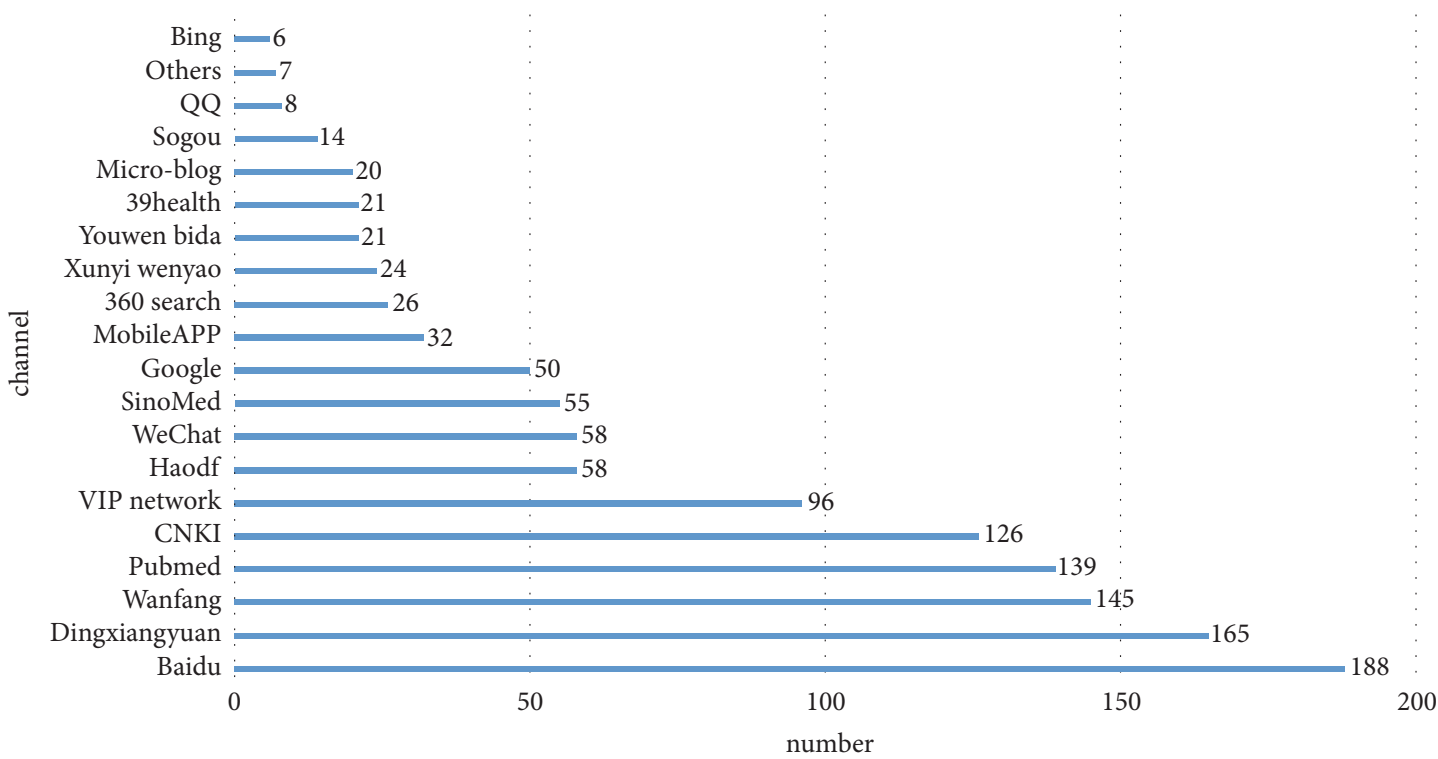

FIGURE 2: The approaches by doctors to acquire medical knowledge by searching the Internet.

\section{Results}

3.1. General Information. A total of 292 valid questionnaires were collected from the respondents, including 134 males and 158 females, with ages ranging from 18 to 50 . Of the respondents, all were clinicians with 173 who had the educational level of a master's degree or above, accounting for $59.24 \%$ of all respondents. They covered all major clinical departments of the hospital. Their titles were evenly distributed from residents to chief physician. According to the monitoring results of the respondents' IP addresses, the geographic distribution of the respondents covered most areas of China, with the most respondents being in Beijing. All questionnaires were submitted by mobile phone. Table 1 shows the demographic details of the respondents.

3.2. Investigation of the Status of Acquiring Professional Medical Knowledge via the Internet in the Doctors. In this study, more than half of the doctors (59.93\%) regularly used the Internet to search for medical knowledge; 142 respondents (48.63\%) often used it, and 33 respondents (11.30\%) always used it, as shown in Figure 1. The most popular search engine was Baidu (with a selection rate of $64.38 \%, 188 / 292$ ), and $19.86 \%$ physicians selected WeChat as an approach to acquire specialized knowledge, as shown in Figure 2. Doctors' satisfaction with using the Internet for acquiring medical 
TABLE 1: Summary of general information.

\begin{tabular}{|c|c|c|}
\hline Category & $\begin{array}{l}\text { Number of } \\
\text { respondents }\end{array}$ & Percentage (\%) \\
\hline \multicolumn{3}{|l|}{ Gender } \\
\hline Males & 134 & 45.89 \\
\hline Females & 158 & 54.11 \\
\hline \multicolumn{3}{|l|}{ Age } \\
\hline Under 18 & 0 & 0 \\
\hline $18-25$ & 48 & 16.44 \\
\hline $26-30$ & 61 & 20.89 \\
\hline $31-40$ & 95 & 32.53 \\
\hline $41-50$ & 72 & 24.66 \\
\hline $51-60$ & 15 & 5.14 \\
\hline Above 60 & 1 & 0.34 \\
\hline \multicolumn{3}{|l|}{ Education } \\
\hline High school/vocational school & 2 & 0.68 \\
\hline College/university & 117 & 40.07 \\
\hline Master & 119 & 40.75 \\
\hline Doctorate or above & 54 & 18.49 \\
\hline \multicolumn{3}{|l|}{ Professional level } \\
\hline Intern & 48 & 16.44 \\
\hline Resident & 69 & 23.63 \\
\hline Attending doctor & 84 & 28.77 \\
\hline Associate Professor & 61 & 20.89 \\
\hline Professor & 30 & 10.27 \\
\hline Total & 292 & \\
\hline
\end{tabular}

knowledge was not high. Only $23.97 \%$ of the respondents were satisfied in the overall evaluation of searching the Internet for medical expertise, as shown in Figure 3.

3.3. Survey of WeChat Usage. Regarding the usage of WeChat, 285 respondents accessed WeChat every day, accounting for $97.60 \%$ of the sample. These daily users were classified by the number of times accessing WeChat, with an interval of 10 . The number of respondents who accessed it 1-10 times per day was the highest, accounting for $32.28 \%$ (92/285), followed by respondents who accessed it 10-20 times, accounting for $29.47 \%(84 / 285)$. Notably, the number of doctors who accessed it more than 20 times per day accounted for $38.24 \%$ $(45+27+37=109,109 / 285)$, indicating that the frequency of WeChat usage in Chinese doctors was very high, as shown in Figure 4. In addition, the doctors also spent a long period of time using WeChat. Figure 5 shows that over 70\% (206/285) of the respondents spent more than 30 minutes per day using WeChat.

3.4. Survey of the Situation for Acquiring Professional Medical Knowledge Using WeChat. Nearly half $(47.26 \%, 111+27=$ 138 , and 138/292) of the respondents agreed that they often

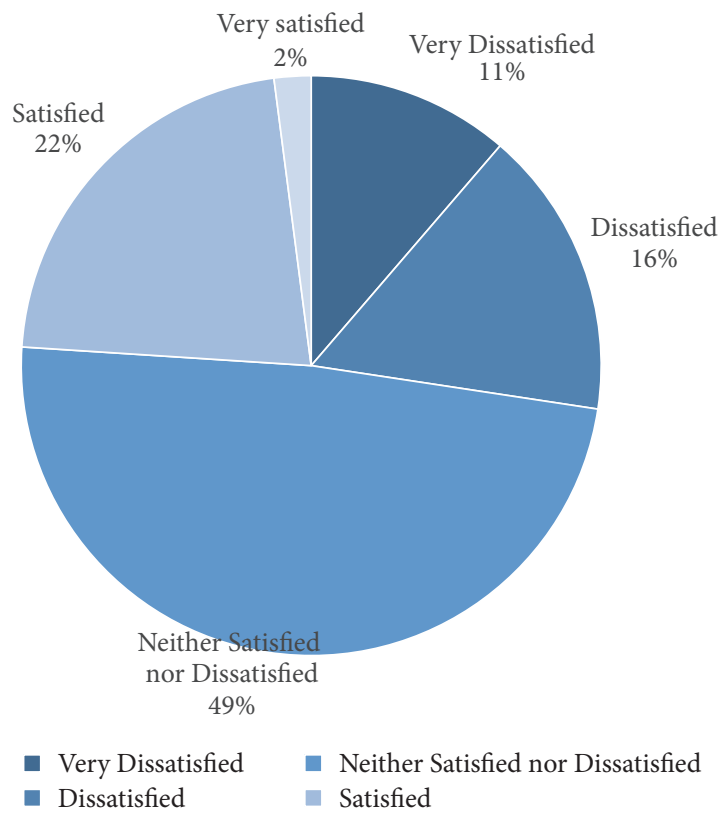

FIGURE 3: Satisfaction with using the Internet to search for medical knowledge.

found medical articles on WeChat, as shown in Figure 6. Additionally, $41.44 \%$ of the respondents said that they regularly accessed professional medical articles on WeChat. When the doctors were searching for medical information on WeChat, the most common methods included browsing friends' moments (with a selection rate of 59.93\%, 175/292) and public account subscriptions (with a selection rate of $60.27 \%, 176 / 292$ ), followed by Dingxiangyuan (an online community for Chinese physicians), WeChat search and group chat, as shown in Figure 7. Readability was the highest in the doctors' assessment of medical knowledge on WeChat, whereas the ratings for professionalism and usefulness were relatively low, as shown in Figure 8. Currently, the potential problems of WeChat with regard to medical expertise of the highest concern for the doctors included the homogenization of information, unguaranteed professionalism, and too many advertisements, with selection rates of $67.81 \%, 66.44 \%$, and $61.30 \%$, respectively. The issue over which there was the least concern was readability. $45.21 \%$ of respondents believed that medical knowledge from WeChat had no help; $36.30 \%$ of respondents were neutral; only $18.50 \%$ believed that WeChat health information could improve professional skills.

3.5. The Most Desirable Means to Acquire Medical Knowledge. Study participants held high expectations for new media like WeChat. In the survey of the most desirable channel by which to acquire medical knowledge through the Internet, Baidu was still the preferred website for searching (with a selection rate of $55.82 \%, 163 / 292$ ), and the selection rate for WeChat was $34.25 \%(100 / 292)$, as shown in Figure 9. Regarding the doctors' mode of acquiring medical knowledge through WeChat, "professional medical knowledge from peers, with a reminder," had the highest score of 80.67 out of 100 points, indicating that professional knowledge posted 


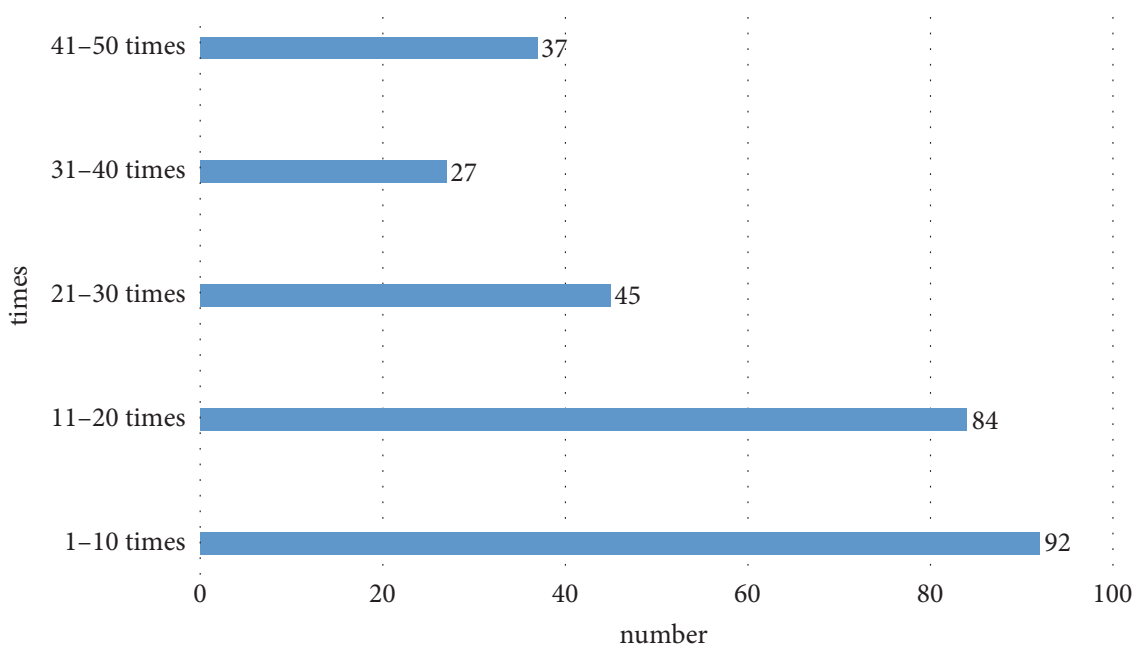

Figure 4: The number of times accessing WeChat per day.

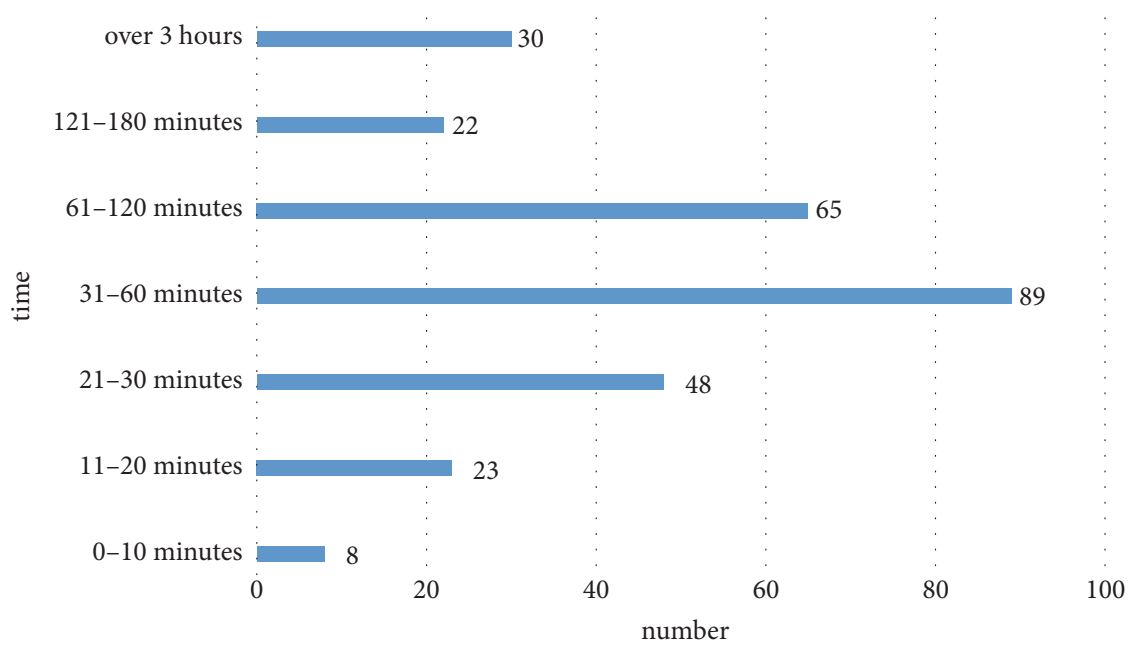

FIGURE 5: Length of time of WeChat usage with the number of users.

through interaction between peers was recognized the most by the doctors. Details were shown in Figure 10.

\section{Discussion}

4.1. WeChat Has Become an Important and Nonnegligible Mobile Supplement for Doctors to Obtain Medical Knowledge. Continuing medical education plays an irreplaceable role in the education of medical professionals. Well-developed continuing medical education and training for healthcare professionals after the completion of their academic education in college can allow them to continuously update their professional knowledge and improve their operational capacity throughout their career, meet the needs of the development of medical science and technology, and enhance the overall level of medical care. Due to the particularity of the contents in continuing medical education, although medical professionals are eager to learn with clear objectives, they have different knowledge backgrounds, and their disposable time is significantly random and fragmented, lacking a long fixed period of time for concentrated study. The effectiveness of the implementation of mobile continuing medical education has not been very satisfactory.

WeChat is a potential way of acquiring professional medical knowledge for busy doctors. As is known to all, many people nowadays use social media as alternative source of information. Because the Internet makes retrieval easy and there are no time and space constraints, it is recognized by the vast majority of doctors and has increasingly become the mode of acquiring medical knowledge with the highest degree of trust in the doctors in the sample. However, only $23.97 \%$ of them were satisfied with acquiring medical knowledge through the Internet. It is noteworthy that, in this study, $19.86 \%$ of the physicians selected the new interactive social media WeChat as the Internet search application for acquiring medical knowledge. Over $70 \%$ of the surveyed physicians spent more than 30 minutes per day on WeChat, occupying an important portion of the doctors' fragmented 


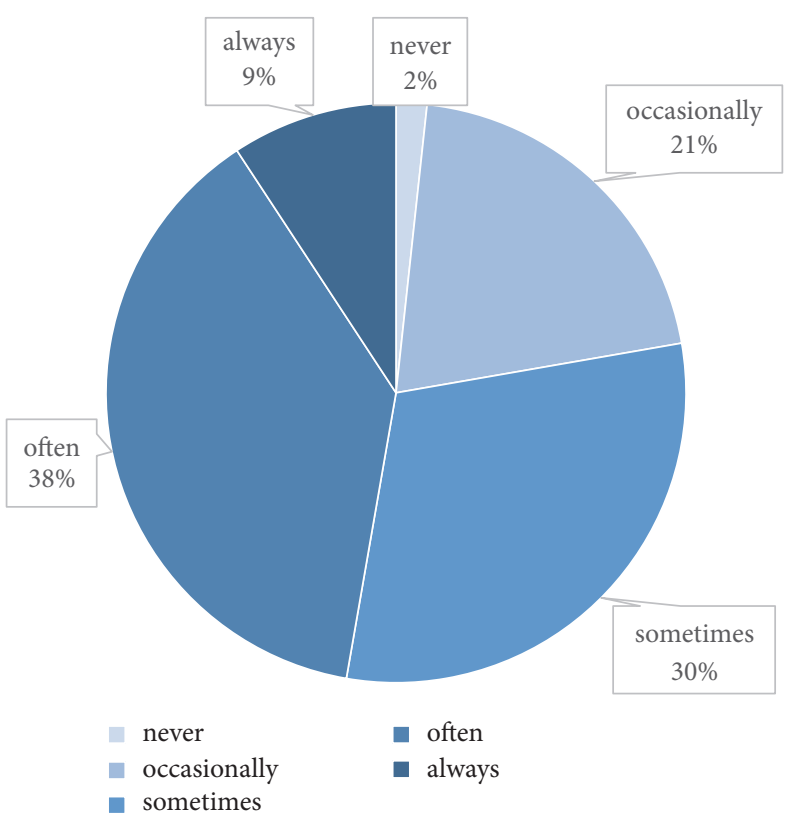

FIGURE 6: Frequency of accessing medical knowledge on WeChat.

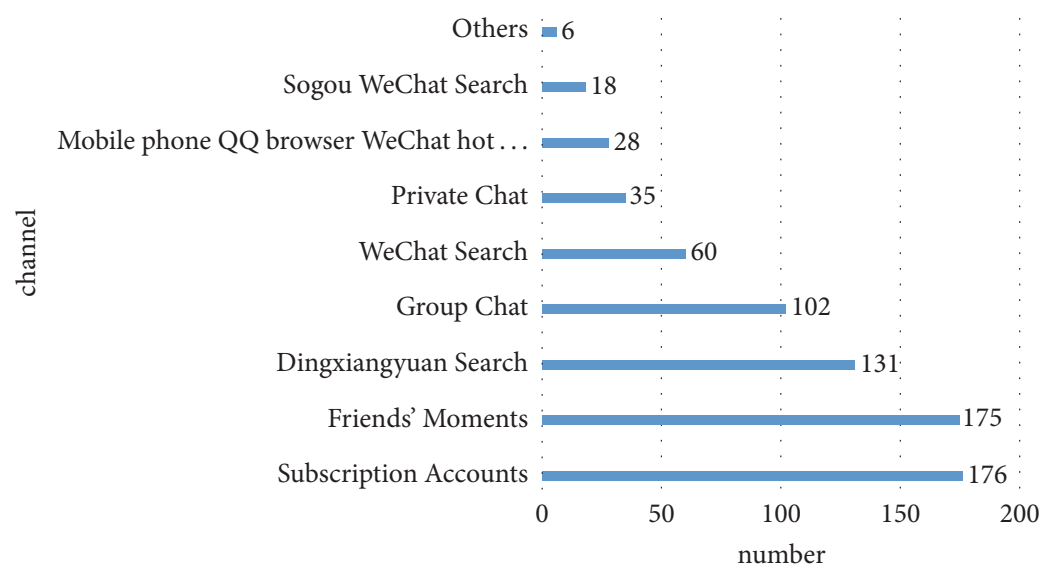

FIGURE 7: Approaches to searching for medical knowledge on WeChat.

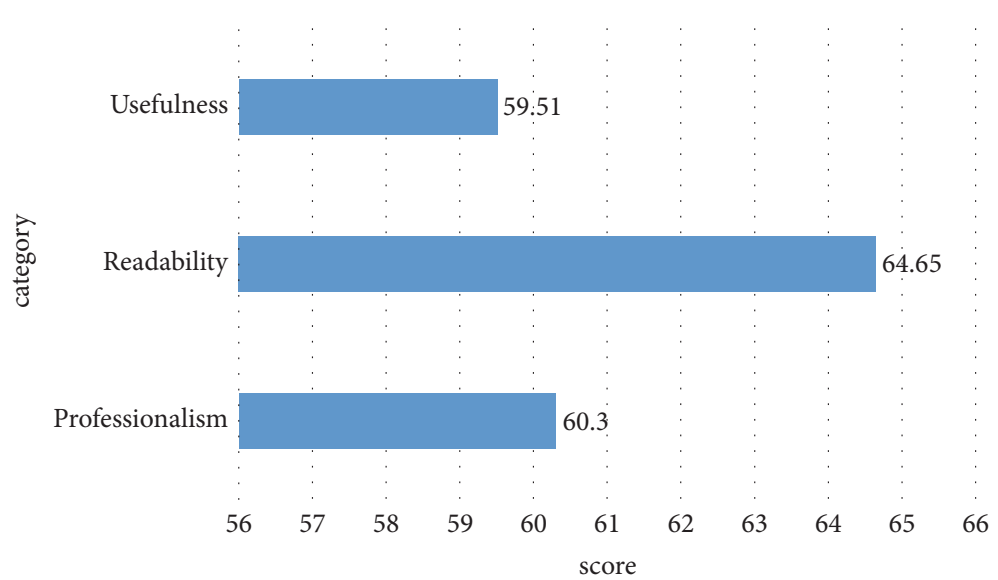

FIGURE 8: Quality assessment of medical knowledge on WeChat by doctors. 


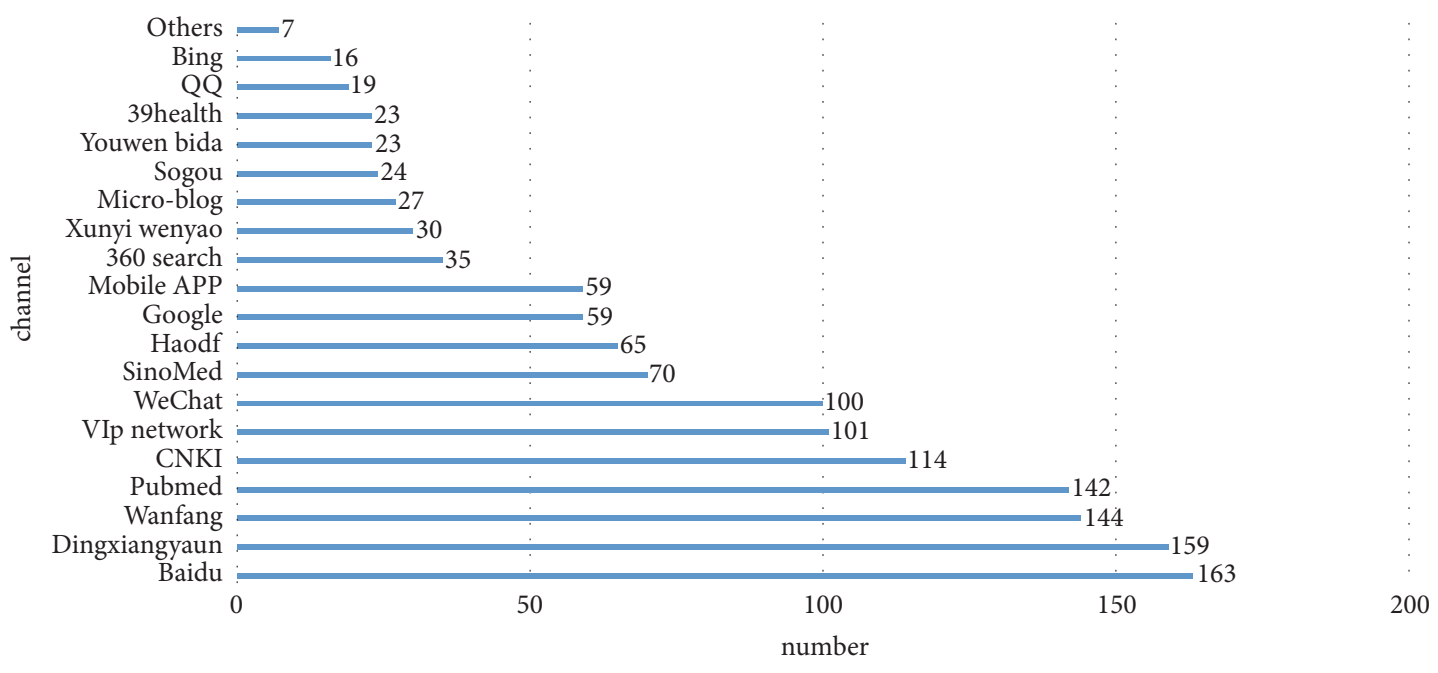

FIgURE 9: The most desirable channels by which to acquire medical knowledge.

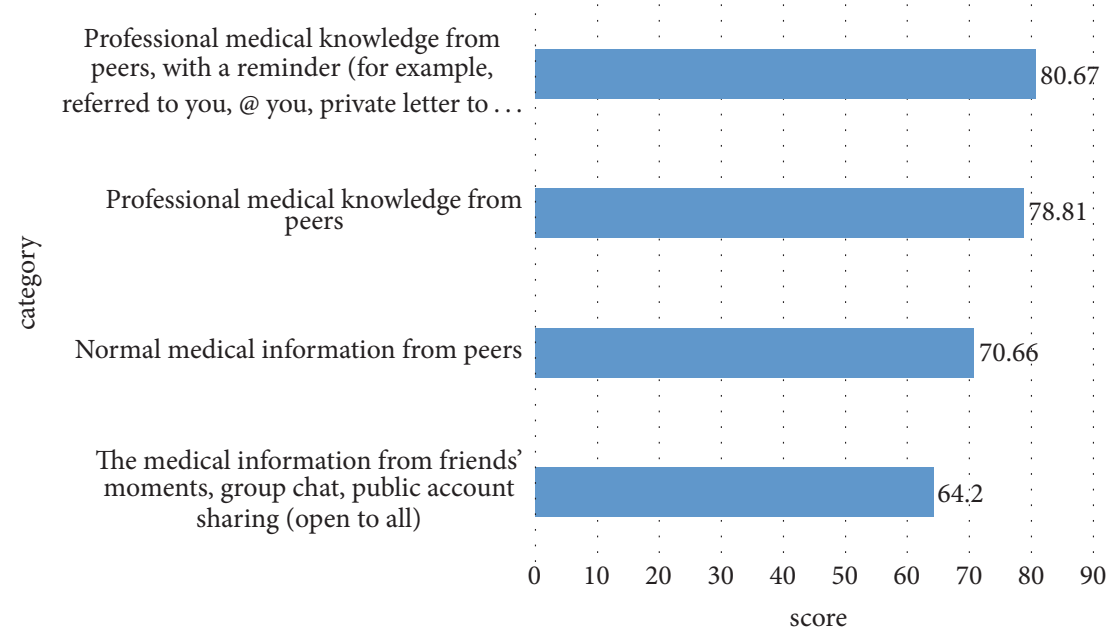

FIGURE 10: Mode for acquiring medical knowledge on WeChat.

time. It has become an important supplement for the doctors to obtain medical knowledge.

Studies have corroborated the feasibility of WeChat as a tool for medical education. The research and practice of Bai et al., who used WeChat to construct a management platform, also confirmed that the use of WeChat not only meets the daily habits of residents but also expands teaching resources to a certain extent, thereby achieving the exchange and sharing of excellent teaching resources [26]. Wang et al. analyzed the application of mobile learning tools in eLearning and indicated that the application of WeChat and Line in mobile learning can enhance the communication of information and contribute to information sharing and problem solving [27]. The study by Li et al. shows that WeChat can improve the quality and capacity of doctors, greatly expand their purview of clinical diseases, and promote the exchange of knowledge of different cases among resident doctors from different departments and disciplines [28].

WeChat and other social media education platforms have been widely recognized by the contents of medical education due to their characteristics related to flexible knowledge dissemination, freedom from the constraints of time and place, the large amount of information, rapid dissemination, the large audience size, ease of management, and strong social attributes and interaction, and they have become an important platform for medical education organizations to perform medical education. Currently, acquiring medical knowledge and the latest medical information through the Internet and new media has become a main means through which active medical staff access information $[29,30]$.

\subsection{Improving Physicians' Awareness of the Various Searching} Methods of WeChat Can Help Them Better Use WeChat to Access Professional Knowledge. Doctors may not be familiar with the various searching methods on WeChat. We found that the approaches of acquiring medical knowledge using WeChat by doctors focused on browsing friends' moments, public account subscriptions, Dingxiangyuan browsing for WeChat, and group chat (with selection rates of $59.93 \%$, $60.27 \%, 44.86 \%$, and $34.93 \%$, resp.). The selection rates for 
WeChat search, Sogou (a third-party search engine) search for WeChat, the mobile phone QQ browser, and WeChat hot articles were relatively low, but these methods are actually able to greatly enhance the efficiency of WeChat search. Thus, it is necessary to strengthen training on the relevant skills, thus providing greater convenience for doctors to take full advantage of WeChat in acquiring sufficient medical knowledge. Information overload and the proliferation of false information are the important issues of new media. How to obtain valuable information from redundant information and how to discern truth from falsehood through independent judgment have become the new challenge for all individuals in the era of new media. Whether the user has the abilities to select, understand, question, assess, create, produce, and speculatively respond to the targeted information, particularly the ability of independent judgment, has become a prerequisite that determines whether the user can benefit from the age of new media [31]. With Chinese doctors, due to the unitary structure of professional knowledge and the relative lack of information technology, it is more difficult for them to obtain valuable information from new media, which seriously affects the effectiveness of new media education. Therefore, it is particularly important to enhance the training level in new media knowledge and to improve the new media literacy of doctors [32].

4.3. Assessing and Improving the Professionalism of Medical Knowledge on WeChat Is the Future Direction for Application and Research. There were major concerns with the quality of medical information on WeChat, especially with regard to content. Doctors acquire medical knowledge on the premise of its quality. Only correct professional knowledge has value in learning. In the assessment of the quality of medical knowledge on WeChat by the doctor respondents, the rating on readability was the highest, whereas the evaluation ratings on professionalism and professional improvement were lower. Currently, the potential problems of medical information on WeChat of the highest concern for the doctors included the homogenization of information, unguaranteed professionalism, and too many advertisements. The issue of the least concern was readability, and $45.21 \%$ respondents considered that it had no significant impact on improving their professional skills. This result indicates that nearly half of the doctors distrusted the medical knowledge on WeChat. The overabundant flood of homogeneous information, advertisements, and unprofessional information without filtering may be the main reasons. In a study from another country, Decamp reached a similar conclusion [33]. Because the current medical knowledge on WeChat is mostly from public accounts, whose operators are mostly not professional authorities, the quality of professional medical knowledge on WeChat cannot be guaranteed, which has become the most worrisome problem in using WeChat as a tool for continuing education for doctors. Therefore, it is essential to evaluate article quality and improve the dissemination of high-quality articles. Due to the high professionalism of medical knowledge and its significant impact on public health $[34,35]$, how to more accurately assess the quality of medical knowledge on WeChat, how to ensure the quality of medical knowledge, and how to promote the dissemination of highquality articles on WeChat warrant further study because these factors will determine the frequency and effectiveness of the future application of WeChat in the continuing medical education of doctors.

4.4. Limitations of This Study. First, the present study used the random snowball sampling method in the survey, and the only respondents were doctors in the private WeChat friends' circle of the researchers. Although the doctors were distributed nationwide and the responses were random and independent, these samples are insufficient to represent all doctors in China. However, they may reflect the situation of acquiring medical knowledge and WeChat usage in the doctors of China to a certain extent. This study is the first largescale study in China. Second, because the questionnaires were distributed online, they were convenient in many aspects including questionnaire publishing, collection, and analysis. However, the questionnaire collection period was short and had a small number of valid responses (only 292); thus, randomness may exist in the responses. These factors most likely caused the bias in the results; thus, this study is only an exploratory attempt in the field. Accurate and more convincing research requires a further expanded sample size, a strictly random method, improvement in the reliability and validity of the questionnaire, and strict control of all the details in the survey so that the results can be more objective and scientific.

4.5. Directions and Trends for Future Study. This study is only a preliminary investigation from the perspective of doctors' acquisition of medical knowledge. Our further research is being conducted as follows: first, a larger and strictly randomized investigation on the channel of continuing education in doctors and the impact of WeChat on continuing education; second, a study of other behaviors and the effects of WeChat usage by doctors, such as research on the doctorpatient interaction and doctors' attitudes toward and approach to online medical consultation service using WeChat; and third, research on quality assessment methods for medical knowledge on WeChat and how to achieve more effective communication for high-quality articles through WeChat. In summary, social media, especially WeChat, have profoundly changed the public's lifestyle and become part of the public's everyday life. WeChat will certainly have an increasingly important impact on healthcare services, such as medical knowledge learning, health education, and medical services in doctors. Research and application in this area will be a very important direction.

\section{Conclusion}

The traditional methods of acquiring medical knowledge have been unable to meet the needs of doctors. The present study shows that WeChat has already become an important mobile means for doctors to acquire medical expertise. Naturally, doctors have not yet fully mastered the approaches to performing searches on WeChat, and the quality of medical knowledge on WeChat is worrisome. Thus, further quality assessment methods are needed to assess and improve the 
quality of medical knowledge on WeChat. These factors preclude doctors from taking advantage of WeChat for professional development. In conclusion, WeChat plays an increasingly important role in acquiring medical knowledge and continuing education for Chinese doctors.

\section{Disclosure}

In addition, this paper was selected as one of the outstanding papers and partially presented in conference CHIP in November 2017.

\section{Conflicts of Interest}

The authors declare that they have no conflicts of interest.

\section{Authors' Contributions}

Jianbo Lei developed the conceptual framework and research protocol for the study. Xingting Zhang and Li Gao conducted the questionnaire creation, distribution, collection, and data analysis. Li Liu and Kunyan Wei interpreted the data and drafted the manuscript. Jianbo Lei made major revisions. All authors approve the final version of the manuscript. Li Liu and Kunyan Wei contributed equally to this work.

\section{Acknowledgments}

This study was partly supported by the National Natural Science Foundation of China (NSFC) (Grant nos. 81471756 and 81771937).

\section{References}

[1] A. P. Abernethy, L. M. Etheredge, P. A. Ganz et al., "Rapid-learning system for cancer care," Journal of Clinical Oncology, vol. 28, no. 27, pp. 4268-4274, 2010.

[2] B. S. McGowan, M. Wasko, B. S. Vartabedian, R. S. Miller, D. D. Freiherr, and M. Abdolrasulnia, "Understanding the factors that influence the adoption and meaningful use of social media by physicians to share medical information," Journal of Medical Internet Research, vol. 14, no. 5, article no. ell7, 2012.

[3] L. Dini, C. Galanski, S. Döpfmer et al., "Online platform as a tool to support postgraduate training in general practice - A case report," GMS Journal for Medical Education, vol. 34, no. 5, Article ID Doc59, 2017.

[4] S. X. Li and R. Pinto-Powell, "Revisiting the merits of a mandatory large group classroom learning format: an MDMBA perspective," Medical Education Online, vol. 22, no. 1, p. 1396174, 2017.

[5] S. Batt-Rawden, T. Flickinger, J. Weiner, C. Cheston, and M. Chisolm, "The role of social media in clinical excellence," The Clinical Teacher, vol. 11, no. 4, pp. 264-269, 2014.

[6] L. Rainie, "A biography of the pew research center's Internet \& American life project," Encyclopedia of Cyber Behavior, vol. 1, pp. 25-41, 2012.

[7] Brenner J, S., Internet \& American Life Project Tracking Survey. Pew Research Center, 2013.

[8] J. M. Alpert and F. E. Womble, "Just What the Doctor Tweeted: Physicians' Challenges and Rewards of Using Twitter," Health Communication, vol. 31, no. 7, pp. 824-832, 2016.
[9] China Internet Network Information Center, the 37th statistic reports on China's Internet development, 2015. http://www .cnnic.cn/hlwfzyj/hlwxzbg/hlwtjbg/201601/t20160122_53271.htm.

[10] Tencent.Inc.Wechat home page. http://www.wechat.com/en/.

[11] Baidu Encyclolpedia, Introduction to WeChat, http://baike .baidu.com/link?url=LLN1376yqNHpkwQKcZfROHtw_bMvbg150T7o4-ou6lMoT2PV3LeT761vREB2JEnYXhUzjhVIod7P4p32O1Cl6DrX9DmpTkCPEQV_d4gc5My.

[12] Curiositychina, statistic report of WeChat users, http://curiositychina.com/.

[13] H. Tong, The broadcast of and impact of WeChat, vol. 09, Chongqing Social Sciences, 2013.

[14] C. He, S. Wu, Y. Zhao et al., "Social Media-Promoted Weight Loss Among an Occupational Population: Cohort Study Using a WeChat Mobile Phone App-Based Campaign," Journal of Medical Internet Research, vol. 19, no. 10, p. e357, 2017.

[15] X. Zhang, D. Wen, J. Liang, and J. Lei, "How the public uses social media wechat to obtain health information in China: A survey study," BMC Medical Informatics and Decision Making, vol. 17, article no. 66, 2017.

[16] B. Cao, C. Liu, M. Durvasula et al., "Social media engagement and HIV testing among men who have sex with men in China: A nationwide cross-sectional survey," Journal of Medical Internet Research, vol. 19, no. 7, article no. e251, 2017.

[17] T. S. O’Hagan, D. Roy, B. Anton, and M. S. Chisolm, "Social Media Use in Psychiatric Graduate Medical Education: Where We Are and the Places We Could Go," Academic Psychiatry, vol. 40, no. 1, pp. 131-135, 2016.

[18] L. N. Ko, J. Rana, and S. Burgin, “Teaching \& Learning Tips 5: Making lectures more "active", International Journal of Dermatology, vol. 57, no. 3, pp. 351-354, 2018.

[19] D. Rozgonjuk, K. Saal, and K. Täht, "Problematic Smartphone Use, Deep and Surface Approaches to Learning, and Social Media Use in Lectures," International Journal of Environmental Research and Public Health, vol. 15, no. 1, p. 92, 2018.

[20] L. Nicolai, M. Schmidbauer, M. Gradell et al., "Facebook groups as a powerful and dynamic tool in medical education: Mixedmethod study," Journal of Medical Internet Research, vol. 19, no. 12, article no. e408, 2017.

[21] L. Sinnenberg, A. M. Buttenheim, K. Padrez, C. Mancheno, L. Ungar, and R. M. Merchant, "Twitter as a tool for health research: A systematic review," American Journal of Public Health, vol. 107, no. 1, pp. el-e8, 2017.

[22] R. C. Chang, H. Lu, P. Yang, and P. Luarn, "Reciprocal Reinforcement Between Wearable Activity Trackers and Social Network Services in Influencing Physical Activity Behaviors," JMIR mHealth and uHealth, vol. 4, no. 3, p. e84, 2016.

[23] F. Gholami-Kordkheili, V. Wild, and D. Strech, "The impact of social media on medical professionalism: A systematic qualitative review of challenges and opportunities," Journal of Medical Internet Research, vol. 15, no. 8, article no. e184, 2013.

[24] N. S. Fogelson, Z. A. Rubin, and K. A. Ault, "Beyond likes and tweets: An in-depth look at the physician social media landscape," Clinical Obstetrics and Gynecology, vol. 56, no. 3, pp. 495-508, 2013.

[25] About sojump. https://www.wjx.cn/html/aboutus.aspx.

[26] Y. Bai, M. Xu, S. Chen, and X. Zhao, Feasibility study of building a cardiovascular teaching platform using WeChat, vol. 3, Northwest Medical Education, 2015.

[27] J. Wang, W. C. W. Yu, and E. Wu, "Empowering Mobile Assisted Social E-Learning: Students' Expectations and Perceptions," World Journal of Education, vol. 3, 2013. 
[28] L. Li, K. Lv, and S. Zhu, Application of WeChat information release system based on mobile terminal in standardized training of burn surgery residents, vol. 5, Northwest Medical Education, 2014.

[29] E. M. Geyer and D. E. Irish, "Isolated to integrated: An evolving medical informatics curriculum," Medical Reference Services Quarterly, vol. 27, no. 4, pp. 451-461, 2008.

[30] E. S. Schwenk, L. F. Chu, R. K. Gupta, and E. R. Mariano, "How Social Media Is Changing the Practice of Regional Anesthesiology," Current Anesthesiology Reports, vol. 7, no. 2, pp. 238245, 2017.

[31] B. M. Walter, R. M. Schmid, and S. Von Delius, "Improving patient information - Are the new media already requested? A questionnaire study at a gastroenterology outpatient clinic," Zeitschrift für Gastroenterologie, vol. 55, no. 6, pp. 551-556, 2017.

[32] J. Salem, H. Borgmann, A. MacNeily et al., "New Media for Educating Urology Residents: An Interview Study in Canada and Germany," Journal of Surgical Education, vol. 74, no. 3, pp. 495-502, 2017.

[33] M. Decamp, "Physicians, social media, and conflict of interest," Journal of General Internal Medicine, vol. 28, no. 2, pp. 299-303, 2013.

[34] Y. Sugawara, H. Narimatsu, A. Hozawa, L. Shao, K. Otani, and A. Fukao, "Cancer patients on Twitter: A novel patient community on social media," BMC Research Notes, p. 699, 2012.

[35] K. C. Chretien, J. Azar, and T. Kind, "Physicians on twitter," Journal of the American Medical Association, vol. 305, no. 6, pp. 566$568,2011$. 


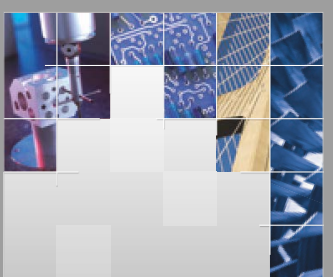

\section{Enfincering}
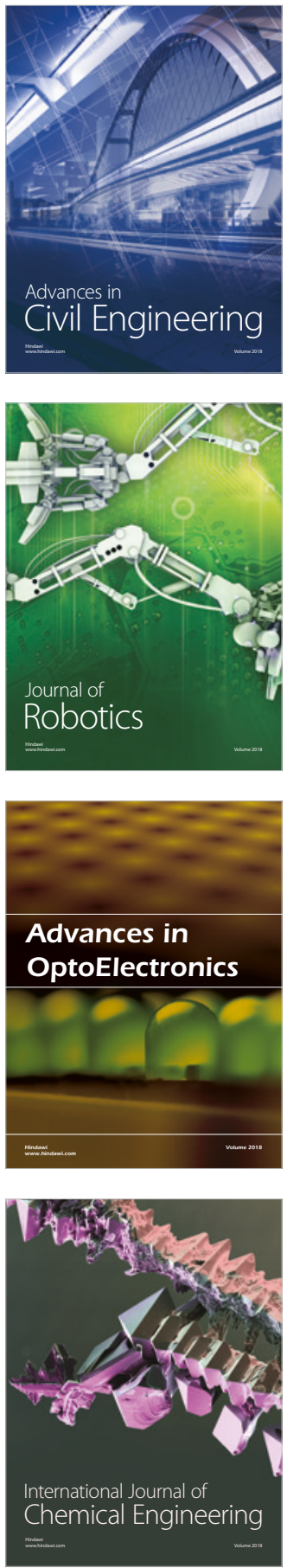

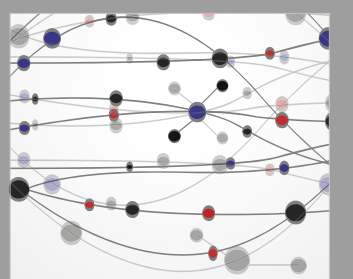

\section{Rotating \\ Machinery}

The Scientific World Journal

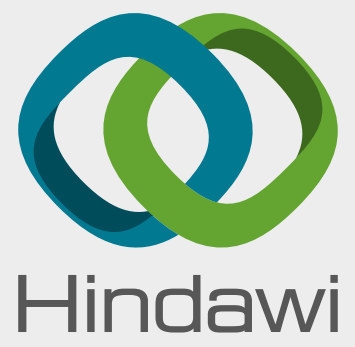

Submit your manuscripts at

www.hindawi.com
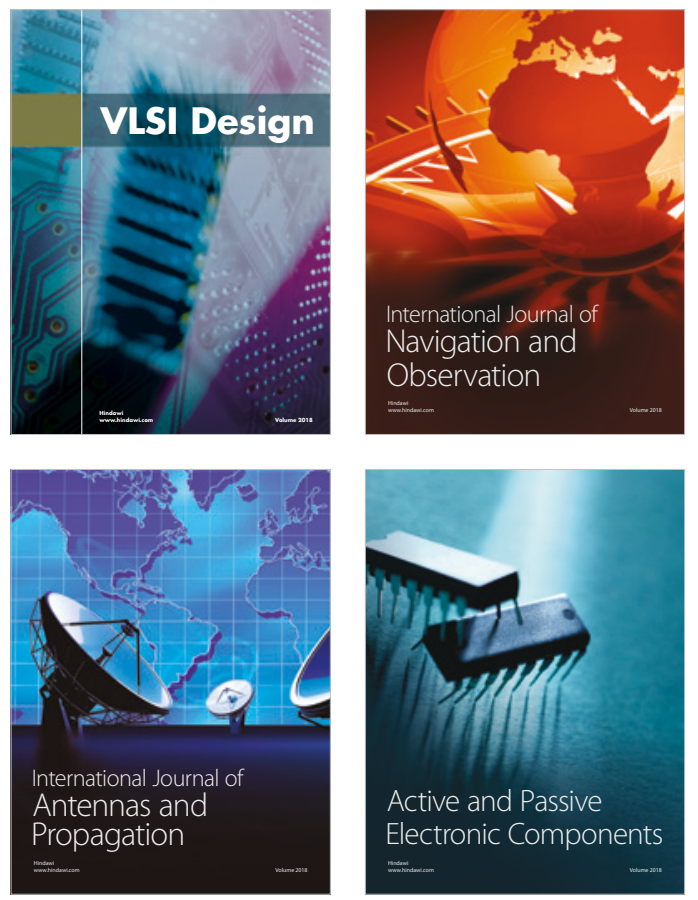
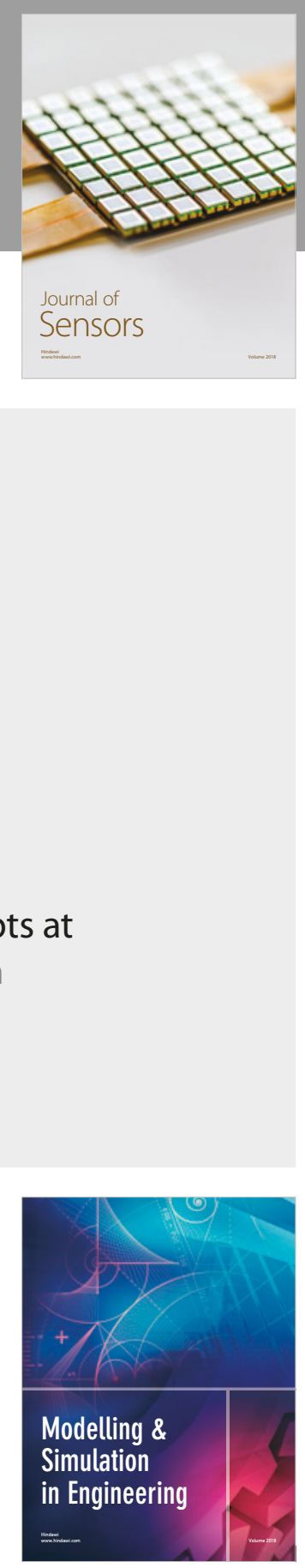

\section{Advances \\ Multimedia}
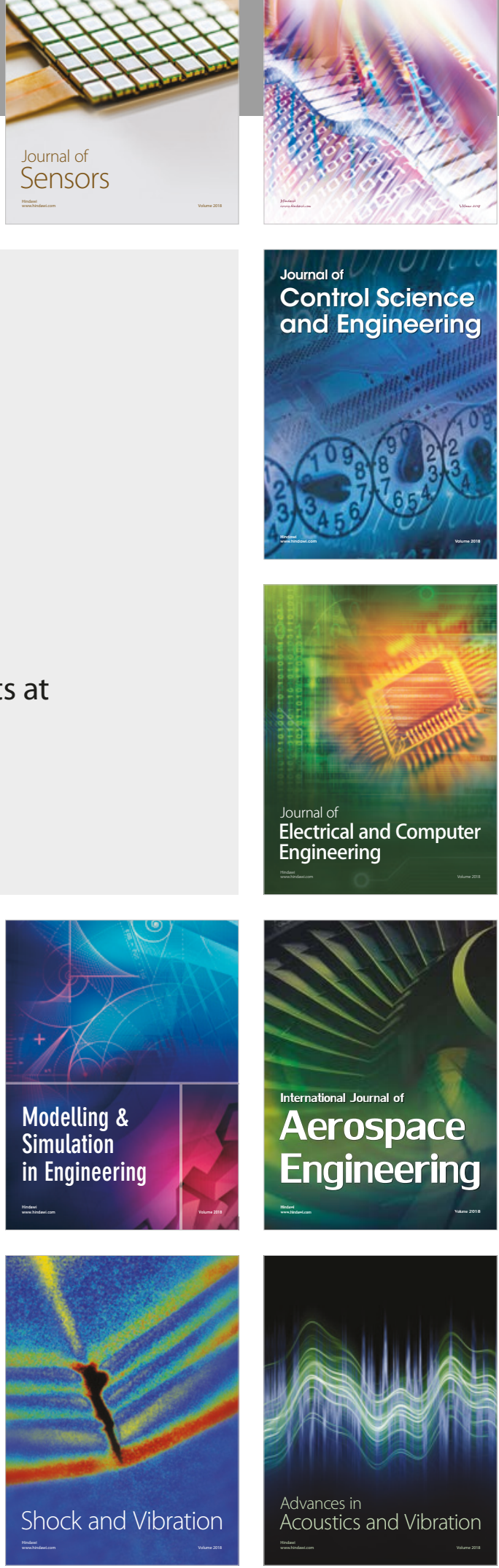